\title{
Arm Recoil Test
}

National Cancer Institute

\section{Source}

National Cancer Institute. Arm Recoil Test. NCI Thesaurus. Code C90496.

A test commonly administered to assess the physiologic response to extending the arms as straight as possible alongside the body when lying down. 